\title{
Psychiatric Assessment and Screening for the Elderly in Primary Care: Design, Implementation, and Preliminary Results
}

\author{
Robert C. Abrams, ${ }^{1}$ Blanca Boné, ${ }^{2}$ M. Cary Reid, ${ }^{2}$ Ronald D. Adelman, ${ }^{2}$ \\ Risa Breckman, ${ }^{2}$ Ronald Goralewicz, ${ }^{3}$ Marlena Palombo, ${ }^{4}$ Amy Stern, \\ Rouzi Shengelia, ${ }^{2}$ and Jeanne Teresi ${ }^{2,6,7}$
}

\author{
${ }^{1}$ Department of Psychiatry and Division of Geriatrics and Palliative Medicine, Weill Cornell Medical College, P.O. Box 140, \\ New York Presbyterian Hospital, 525 East 68th Street, New York, NY 10065, USA \\ ${ }^{2}$ Division of Geriatrics and Palliative Medicine, Weill Cornell Medical College, P.O. Box 39, New York Presbyterian Hospital, \\ 525 East 68th Street, New York, NY 10065, USA \\ ${ }^{3}$ The Irving Sherwood Wright Center on Aging and Department of Nursing, New York Presbyterian Hospital, 1484 First Avenue, \\ New York, NY 10075, USA \\ ${ }^{4}$ The Irving Sherwood Wright Center on Aging and Department of Social Work, New York Presbyterian Hospital, 1484 First Avenue, \\ New York, NY 10075, USA \\ ${ }^{5}$ Weill Cornell Institute of Geriatric Psychiatry and Department of Social Work, New York Presbyterian Hospital, \\ 21 Bloomingdale Road, White Plains, NY 10605, USA \\ ${ }^{6}$ Research Division, Hebrew Home at Riverdale, 5901 Palisade Avenue, Riverdale, NY 10471, USA \\ ${ }^{7}$ Stroud Center, Columbia University, New York State Psychiatric Institute, New York, NY 10032, USA
}

Correspondence should be addressed to Robert C. Abrams; rabrams@med.cornell.edu

Received 31 January 2015; Accepted 5 March 2015

Academic Editor: Iracema Leroi

Copyright (C) 2015 Robert C. Abrams et al. This is an open access article distributed under the Creative Commons Attribution License, which permits unrestricted use, distribution, and reproduction in any medium, provided the original work is properly cited.

\begin{abstract}
Introduction. We describe the design and implementation of a psychiatric collaborative care model in a university-based geriatric primary care practice. Initial results of screening for anxiety and depression are reported. Methods and Materials. Screens for anxiety and depression were administered to practice patients. A mental health team, consisting of a psychiatrist, mental health nurse practitioner, and social worker, identified patients who on review of screening and chart data warranted evaluation or treatment. Referrals for mental health interventions were directed to members of the mental health team, primary care physicians at the practice, or community providers. Results. Subjects $(N=1505)$ comprised $38.2 \%$ of the 3940 unique patients seen at the practice during the 4-year study period. $37.1 \%(N=555)$ screened positive for depression, $26.9 \%(N=405)$ for anxiety, and $322(21.4 \%)$ screened positive for both. Any positive score was associated with age $(P<0.033)$, female gender $(P<0.006)$, and a nonsignificant trend toward living alone $(P<0.095) .8 .87 \%$ had suicidal thoughts. Conclusions. Screening captured the most affectively symptomatic patients, including those with suicidal ideation, for intervention. The partnering of mental health professionals and primary care physicians offers a workable model for addressing the scarcity of expertise in geriatric psychiatry.
\end{abstract}

\section{Introduction}

Spurred by the high frequency of psychiatric symptoms in geriatric primary care populations, particularly depression and anxiety [1-4] and their associated adverse consequences, mental health services have recently been brought directly to the settings in which elderly patients are found [5-9].
The services that provide psychiatric treatment within primary care practices are known as collaborative care models (CCMs). CCMs address a hitherto unmet need in geriatric mental health. While a majority of older adults receive health care from primary providers, most front-line physicians are not psychiatrically trained and do not provide mental health services $[10,11]$. 
CCMs offer several key advantages [12]. CCMs can reduce the stigma surrounding psychiatric disorders in the elderly [13], ease access to expert psychopharmacological treatment [14], and improve care coordination between mental health and primary care teams $[13,14]$. Large-scale trials using CCMs to manage older adults with depression or anxiety have reported encouraging results [7-9], although sustainability in the various settings has not been firmly established $[15,16]$.

In this paper we describe the design and implementation of a new CCM model co-located with and integrated into a university-based ambulatory geriatric primary care practice. The service, denoted by the acronym PASSE-PC (Psychiatric Assessment and Screening for the Elderly-Primary Care), began as a demonstration project at Weill Cornell Medical College in New York City, funded by the New York State Office of Mental Health (OMS). Weill Cornell was part of a multicenter OMS research initiative sponsoring the integration of mental health services within a broad range of medical settings and patient populations.

A central feature of PASSE-PC is case-finding that uses on-site screening for symptoms of depression and anxiety, followed by targeted psychiatric treatment when indicated. Here we also report data from the initial screenings of 1505 older persons as well as comparisons of the clinical, demographic, and psychosocial features of screen-positive and screen-negative patients.

\section{Materials and Methods}

2.1. Study Site. The study was conducted at the Irving S. Wright Center on Aging, the outpatient group practice of the Weill Cornell Medical College's Division of Geriatrics and Palliative Medicine. The practice provides primary care to an older patient population (mean age in the mid-80s) that is mostly medicare-insured, female, and non-Hispanic white. The majority of the center's patients live independently or semi-independently with home supervision; approximately $10 \%$ reside in assisted-living facilities. The study was approved by the Institutional Review Board of the Weill Cornell Medical College.

2.2. Subject Inclusion and Exclusion Criteria. Consistent with requirements for enrollment at the Wright Center, subjects were considered for inclusion if they were 50 years of age or older and were receiving, or planned to receive, their primary medical care at the practice. Excluded were patients who, based on clinical impression, had sensory or cognitive impairments severe enough to preclude meaningful participation in screening for anxiety or depression.

\subsection{Logistics of Screening and Team Meetings}

2.3.1. Time Frame. All screening for depression and anxiety took place from April 2008 through June 2012, the period coinciding with funding support for the project.

2.3.2. Protocol for Screening New Patients. Included in the registration packet for patients new to the center was a single page, with a screening instrument for depression, the PHQ9 (Patient Health Questionnaire-9) [17-21], on one side and a screening instrument for anxiety, and the GAD-7 (Generalized Anxiety Disorder-7) [22], on the reverse side, both printed in large, bold font. Patients were asked to circle the number corresponding to the severity and/or frequency of symptoms described in each item. The completed forms were then presented to the patient's PCP (Primary Care Physician) during the visit. The PCP entered the questionnaire responses in an addendum to the electronic medical record. Patients who experienced difficulty because of visual impairment, cognitive deficits, or a language barrier were assisted by a family member or volunteer. Translators were available with advance notice. If for any reason the patient had not completed the questionnaire, the PCP was tasked to help him or her do so at the time of the examination.

2.3.3. Protocol for Screening Established Patients. Patients already receiving primary care at the center who had not been previously screened (or were due to be rescreened) were identified by the mental health team from the list of appointments scheduled daily. These individuals were given the page containing the PHQ-9 and GAD-7 at the reception desk. The patients recorded their responses in the waiting room and then handed off the forms to the PCP.

2.3.4. Frequency of Screening. Patients with positive initial screens (PHQ-9 score > 5 and/or GAD-7 score > 5) were rescreened every three months or at the next medical visit closest to a 3-month interval. Patients with negative initial screens were rescreened yearly, or sooner at the discretion of the PCP.

2.3.5. Team Composition and Meetings. The mental health team was composed of a part-time (20\% full time equivalent) geriatric psychiatrist, fulltime psychiatric nurse practitioner (NP), and a fulltime clinical social worker (SW). Mental health team meetings to review screens completed during the previous 5 working days were held weekly. After entering screens into the electronic medical record, the PCPs flagged these data to the attention of the NP or SW for review at the next team meeting.

Team meetings were approximately 90 minutes in length (range 60-120 minutes), during the course of which the screens and medical records of 10 to 20 subjects were reviewed; the actual number of subjects assessed in this way varied according to the weekly patient volume. In each case, including those screening negative, the team reviewed the questionnaire responses and salient features of the individual's clinical history as key steps in determining the patient's needs. The patients triaged for further evaluation and treatment included those with positive scores on one or both instruments, some patients who had not been screened but whose PCP had referred them for assessment, and others with negative scores who were determined from information in the chart to warrant further evaluation or possible treatment. 
The team's recommendations took into account multiple factors, including the severity of depression or anxiety, suicidality, nonaffective psychiatric disorders, cognitive impairment, medical burden, the likelihood that pharmacological treatment would be required, the need for social or psychotherapeutic interventions, any suspicion of elder abuse, and the ability of the patient to adhere to treatment. The validated PHQ-9 scores corresponding to mild, moderate, moderately severe, and severe depression [17-21] were used as guidelines but not followed rigidly in formulating treatment recommendations.

A chart note describing each patient's treatment plan was created and forwarded electronically to the PCP, accompanied by a disclaimer that the mental health team had completed an assessment based upon chart review, but had not interviewed the patient. In most cases, an in-person discussion of the team's recommendations was held with the PCP during the same week.

The principal triage or referral options included (1) full evaluation by a mental health team member; (2) referral to the team's psychiatrist or NP; (3) referral to the team's SW; (4) continued psychopharmacological treatment by the PCP; (5) continued treatment by a community psychiatrist; and (6) referral to a community provider. Some patients were referred to multiple mental health providers or treatment modalities. For example, an individual with major depression and a concurrent housing or financial problem might be referred to both the team's psychiatrist or NP and to its SW. If a patient had been receiving treatment with a mental health professional in the community, the patient's preference to continue that treatment was respected, and liaison with the community-based provider was offered.

2.3.6. Treatments Employed. At the center, treatment by the psychiatrist typically entailed the prescription and monitoring of psychopharmacological agents. Patients assigned to the NP or SW for psychotherapy were treated using a PSP (Problem-Solving Therapy) protocol adapted for use in older patients with affective disorders, including those with executive dysfunction or mild cognitive impairment [23].

2.3.7. Educational Features. If the patient's PCP was to be the principal provider of the psychiatric treatment, he or she was scheduled to receive clinical supervision by a member of the mental health team. Supervisory meetings with the PCPs assuming direct responsibility for mental health treatment were arranged to coincide as closely as possible to the patients' scheduled appointments. The supervisory sessions were focused on clinical case material, but their larger purpose was to help attending and trainee geriatricians develop skills in the diagnosis and treatment of geriatric mood disorders. Particularly challenging patients were selected for discussion with the full center staff using a case conference format led by the geriatric psychiatrist. In addition, the NP and SW themselves received specialized training and supervision in the PST protocol by clinicians with expertise in this type of treatment.
2.4. Data Entry and Management. A database was created that included a unique identification number for each subject; demographics (e.g., age, gender, insurance status); dates of screening and rescreening; PHQ-9 and GAD-7 scores; and disposition. For each of its institutional participants the OMS research program required a second database featuring a needs assessment. The needs assessment documented patient-level factors associated with anxiety and depression, including other mental health problems; cognitive impairment; poor physical health; suicidality; substance abuse; selfneglect; social isolation; housing, financial, and legal issues; and elder abuse. These needs assessments were scored by members of the mental health team using a consensus process after reviewing all available sources of information in each case-that is, the medical chart, screening data, and any other information provided by the PCP. Needs areas were rated as either as absent or as present to a high, moderate, or low level. Another score was given for each of the identified needs, indicating categorically whether or not referrals addressing each need were made.

2.5. Statistical Analysis. Descriptive statistics were calculated for all study variables. We also examined associations between key independent variables (e.g., age, gender) and screen results using bivariate correlations. Logistic regression models were constructed to identify predictors of subjects screening positive for depression and/or anxiety. The first model included gender, race/ethnicity (non-Hispanic white versus other), living status (lives alone versus with others), and age. The second model retained these variables and added mental health and physical health factors elicited for the needs assessments. Colinearity diagnostics were performed and were acceptable.

Two sets of analyses were used to compare scores of treatment groups. First, ANOVAs (analyses of variance) were performed examining group differences separately for anxiety and depression. However, because key variables were correlated, that is, PHQ-9 and GAD-7 were correlated: $0.622(P<$ 0.001 ), a MANOVA (multivariate analysis of variance) was also performed. The ANOVA and MANOVA models were used with Scheffe and Bonferroni post hoc tests to examine the differences between subjects receiving no treatment and subjects receiving each of 4 different modalities of treatment.

To test the hypothesis that within the group of treated subjects, those with higher initial PHQ-9 and GAD-7 scores were more likely to receive medication as part of their treatment, similar Scheffe and Bonferonni post hoc comparisons were made between the Psychotherapy Only and Medications Only groups and also between the Social Services Intervention Only and Medications Only groups.

In addition, bivariate correlations were used to compare PHQ-9 and GAD-7 scores of patients receiving no treatment with those receiving, for this analysis, each of 5 different modalities of treatment. Bivariate correlations were also used to identify relationships between screen positivity and items scored as present on the needs assessment. 


\section{Results}

3.1. Screening. The total number of subjects screened during the study period was 1505 , comprising $38.2 \%$ of the 3940 unique patients seen at the practice during that time. Those not screened included patients not meeting inclusion criteria, subjects excluded from the study, refusals, and those who for logistical or staffing reasons were not asked to participate. The data presented here are from subjects' initial screenings.

3.2. Demographic Characteristics of Overall Group. The mean age of screened subjects was 78.67 (SD = 9.86) years. Although inclusion criteria allowed for subjects as young as 50 years of age, the study population was overwhelmingly geriatric, with only 110 of 1505 screenees $(7.3 \%)$ aged younger than 65 . Most subjects were women $(73.1 \%)$, non-Hispanic white $(81.6 \%)$, relied on Medicare and supplemental coverage (versus Medicaid) (88.0\%), and lived alone (55.8\%).

3.3. Number of Subjects Screening Positive. $37.1 \%(N=558)$ of the patients screened positive for depression, $26.9 \%(N=$ $405)$ screened positive for anxiety, and $21.4 \%(N=322)$ screened positive for both depression and anxiety.

3.4. Demographics of Screen-Negative and Screen-Positive Subjects. Table 1 shows that screening positive was significantly associated with age $(P<0.033)$ and female gender $(P<$ $0.006)$. The association of screening positive and living alone trended toward significance $(P<0.095)$.

3.5. Subjects' $P H Q-9$ and GAD-7 Mean Dimensional Scores. The mean score for the PHQ-9 $(6.84, \mathrm{SD}=5.932$, range $0-27)$ surpassed the cut-off point of 5 for screening positive, while the mean score for the GAD-7 $(4.79, \mathrm{SD}=5.173$, range $0-24)$ closely approached the same cut-off point.

3.6. Predictors of Screening Positive for Depression and/or Anxiety. In the logistic regression model, female gender and age were independent predictors of screening positive for depression and/or anxiety. Females were significantly more likely than males to screen positive for depression and/or anxiety $(\mathrm{OR}=1.371, P=0.021)$, and, as age increased, the likelihood of screening positive also increased $(\mathrm{OR}=1.012$, $P=0.036$ ) (Table 2).

3.7. Proportions of Subjects Screening Negative and Positive Who Were Referred for Treatment. $11.8 \%(N=74)$ of the 627 subjects screening negative were referred for treatment despite their status. A relatively small proportion of the 601 subjects screening positive, less than one-third [31.5\%. $(N=$ 202)], were referred for treatment. However, this referral rate did not include the $28.1 \%(N=169)$ of subjects screening positive who were concurrently receiving treatment from a member of the mental health team, community provider, or Wright Center PCP; nor the 6.5\% $(N=39)$ of subjects screening positive who were referred but refused intervention; nor subjects who were referred directly to treatment by their PCPs but who had not been screened $(N=101)$.
TABLE 1: Demographic characteristics of screen-negative and screen-positive subjects* .

\begin{tabular}{lccccc}
\hline \multicolumn{5}{c}{ Screened for depression and/or anxiety } \\
& \multicolumn{2}{c}{ Negative } & \multicolumn{2}{c}{ Positive } & $P$ value \\
& $N$ & $\%$ & $N$ & $\%$ & \\
\hline Gender & & & & & \\
$\quad$ Male & 190 & 29.1 & 139 & 22.4 & 0.006 \\
$\quad$ Female & 463 & 70.9 & 481 & 77.6 & \\
Race & & & & & \\
$\quad$ Other & 127 & 19.4 & 108 & 17.4 & \\
$\quad$ White & & & & & 0.351 \\
$\quad$ Non- & 526 & 80.6 & 512 & 82.6 & \\
$\begin{array}{l}\text { Hispanic } \\
\text { Has Medicaid }\end{array}$ & & & & & \\
$\quad$ No & 470 & 86.9 & 454 & 89.0 & 0.286 \\
$\quad$ Yes & 71 & 13.1 & 56 & 11.0 & \\
Lives alone & & & & & \\
$\quad \begin{array}{l}\text { No } \\
\text { Yes }\end{array}$ & 293 & 46.1 & 252 & 41.4 & 0.095 \\
$\begin{array}{l}\text { Age in years } \\
(n, \text { mean, s.d. })\end{array}$ & $627,78.02(9.98)$ & $601,79.24(10.01)$ & 0.033 \\
\hline
\end{tabular}

${ }^{*}$ Chi-square tests were used to compare dichotomous variables and $t$-test for age, a continuous variable.

3.8. Comparison of Groups Based on Treatment Modality of the Referrals. Table 3 shows that differences in screening scores between subjects receiving no treatment and those receiving any of 4 categories of treatment ranged from 3.3 to 5.3 points on the PHQ-9 and 3.3 to 4.4 points on the GAD-7. Subjects not referred to treatment were less symptomatic than those who were referred, that is, had significantly lower mean scores on the PHQ-9 and GAD-7 ( $P$ values ranging from 0.001 to 0.039 using the Scheffe post hoc test, and $P$ values ranging from 0.001 to 0.015 using the Bonferroni post hoc test). However, Scheffe and Bonferonni post hoc comparisons made within the group of subjects referred for treatment, that is, between the Psychotherapy Only and Medications Only groups and between the Social Services Intervention Only and Medications Only groups, did not reveal significant differences (table not shown; see Supplementary Material available online at http://dx.doi.org/10.1155/2015/792043).

3.9. Correlations of Treatment Modality and PHQ-9 and GAD7 Scores. Consistent with the post hoc analyses, not being referred for treatment was negatively correlated with participants' PHQ-9 and GAD-7 scores. Thus, subjects not referred for treatment had lower scores or were less symptomatic than those referred for treatment of any kind (correlations were examined using 5 treatment groups for this analysis) (Table 4). Conversely, being referred for treatment of any kind was positively correlated with PHQ-9 and GAD-7 scores. All of these outcomes were significant or trended towards significance. 
TABLE 2: Results of logistic regressions predicting positive screens for depression and/or anxiety* .

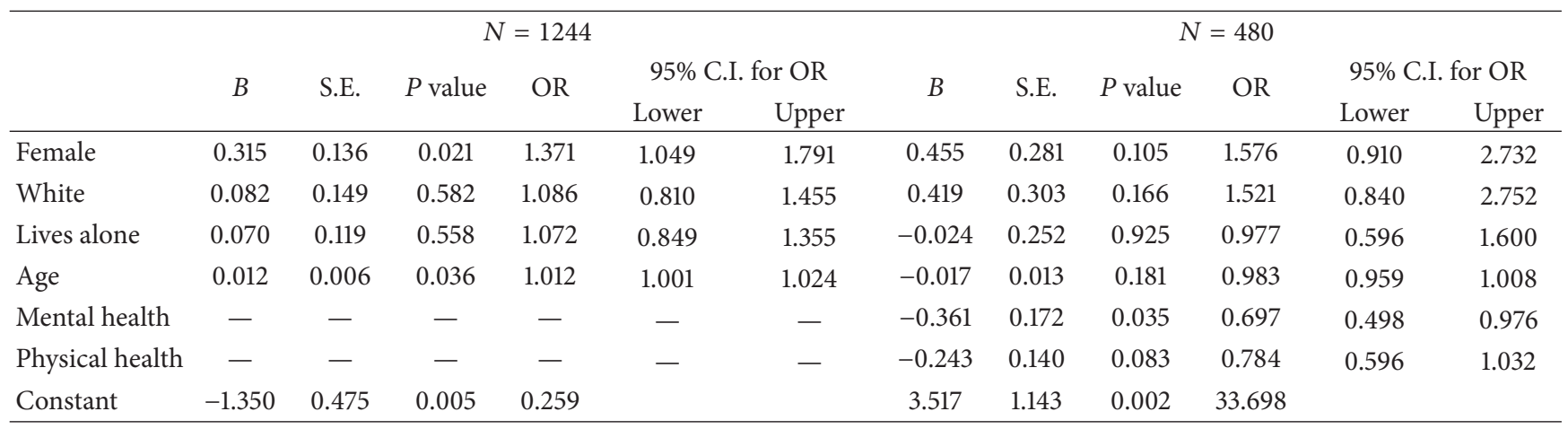

${ }^{*}$ Female, white, and lives alone are dichotomous variables. Age is a continuous variable.

TABLE 3: Summary of Bonferroni post hoc results for PHQ-9 and GAD-7. (All comparisons are against the "No treatment" group).

\begin{tabular}{|c|c|c|c|c|c|c|c|c|c|c|c|c|}
\hline & \multicolumn{6}{|c|}{ PHQ-9 } & \multicolumn{6}{|c|}{ GAD-7 } \\
\hline & \multicolumn{3}{|c|}{ ANOVA } & \multicolumn{3}{|c|}{ MANOVA } & \multicolumn{3}{|c|}{ ANOVA } & \multicolumn{3}{|c|}{ MANOVA } \\
\hline & $\begin{array}{c}\text { Mean } \\
\text { difference }\end{array}$ & $\begin{array}{l}\text { Std. } \\
\text { error }\end{array}$ & $P$ value & $\begin{array}{c}\text { Mean } \\
\text { difference }\end{array}$ & $\begin{array}{l}\text { Std. } \\
\text { error }\end{array}$ & $P$ value & $\begin{array}{c}\text { Mean } \\
\text { difference }\end{array}$ & $\begin{array}{l}\text { Std. } \\
\text { error }\end{array}$ & $P$ value & $\begin{array}{c}\text { Mean } \\
\text { difference }\end{array}$ & $\begin{array}{l}\text { Std. } \\
\text { error }\end{array}$ & $P$ value \\
\hline Medications Only & -4.656 & 0.570 & $<0.001$ & -4.87 & 0.585 & $<0.001$ & -3.402 & 0.506 & $<0.001$ & -3.32 & 0.517 & $<<0.001$ \\
\hline $\begin{array}{l}\text { Psychotherapy } \\
\text { Only }\end{array}$ & -3.328 & 1.137 & $0.035^{*}$ & -3.40 & 1.162 & $0.035^{*}$ & -4.365 & 1.029 & $<0.001$ & -4.29 & 1.028 & $<0.001$ \\
\hline $\begin{array}{l}\text { Social Services } \\
\text { Intervention Only }\end{array}$ & -4.028 & 1.237 & 0.012 & -3.94 & 1.269 & 0.020 & -3.648 & 1.124 & 0.012 & -3.58 & 1.122 & 0.015 \\
\hline $\begin{array}{l}\text { Medications and } \\
\text { Psychother- } \\
\text { apy/Medications, } \\
\text { Psychotherapy, and } \\
\text { Social Services } \\
\text { Intervention }\end{array}$ & -5.328 & 0.820 & $<0.001$ & -5.15 & .846 & $<0.001$ & -4.261 & 0.741 & $<0.001$ & -4.34 & 0.749 & $<0.001$ \\
\hline
\end{tabular}

TABLE 4: Correlations of treatment modality with PHQ-9 and GAD7 scores.

\begin{tabular}{lcc}
\hline & PHQ-9 & GAD-7 \\
\hline No treatment & $-0.339^{* * *}$ & $-0.312^{* * *}$ \\
Medications Only & $0.236^{* * *}$ & $0.190^{* * *}$ \\
Psychotherapy Only & 0.065 & $0.115^{* *}$ \\
Social Services Intervention Only & $0.078^{*}$ & $0.083^{*}$ \\
Medications and Psychotherapy & $0.145^{* * *}$ & $0.126^{* * *}$ \\
Medications, Psychotherapy, and & $0.114^{* *}$ & $0.109^{* *}$ \\
Social Services Intervention & & \\
\hline
\end{tabular}

${ }^{*} P<0.05 ;{ }^{* *} P<0.01 ;{ }^{* * *} P<0.001$.

However, two of the categories involving treatment with medications, namely, Medications Only and Medications and Psychotherapy, had correlations with PHQ-9 and GAD-7 that were significant at the highest level $(P<0.001)$, while treatments involving Social Services Intervention or Psychotherapy, either alone or in combination with other treatments, had correlations with PHQ-9 and GAD-7 that were at lower levels of significance (Table 4).

3.10. Correlation Coefficients for Needs Assessments and Screen-Positive Status. Based on the needs assessments, general mental health issues $(P=0.027)$, cognitive issues $(P=$ $0.075)$, and social isolation $(P=0.006)$, as well as social isolation-generated referrals $(P=0.001)$, were related to screen positivity for depression and/or anxiety. However, only social isolation and social isolation referrals were significant $(P=0.010)$ after adjustment for multiple comparisons (data not shown).

3.11. Prevalence of Suicidality. Scores for the PHQ-9 suicide item, reflecting how frequently suicidal thoughts or impulses were experienced by the subject ( 1 the least often, 4 the most often), were distributed as follows, item score: number of respondents (\%): 1: 52 (3.5\%); 2: 27 (1.8\%); 3: 18 (1.2\%); and $4: 1(0.1 \%)$, for a total positive-score prevalence of $8.87 \%$. 
There were no reported suicide attempts nor completed suicides among the study subjects during the investigational period.

\section{Discussion}

4.1. Review of Findings from Screening for Depression and Anxiety. A principal aim of the study was to determine whether screening for anxiety and depression would be feasible under the PASSE-PC model, useful in identifying cases and manageable with available "in house" and community resources. We had earlier found that the experience of being screened was itself well-accepted by the patients $[24,25]$. Further, we were able, without employing research assistants dedicated to the project and therefore avoiding additional expenditure, to screen over one-third of the unique individuals presenting to the center during the study period.

The center's location in a relatively high-income and racially homogeneous area (the Upper East Side of Manhat$\tan$ ), resulted in a sample that was mostly non-Hispanic white and had a low rate of Medicaid coverage. The overrepresentation of females also reflected the center's demographics. We did not measure ability to perform ADLs (activities of daily living) nor did we use screening scores for cognitive status, but these community-dwelling patients rarely presented with severe functional limitations or advanced dementia, and those who had the latter were, as noted, excluded from the study on clinical-operational grounds if they were unable to participate in screening for depression and anxiety. Otherwise, issues around subjects' functional status, cognitive impairment, drug and alcohol use, and social problems were considered broadly in clinical and needs assessments but not specifically quantified for this data set.

The clinical yield from screening was substantial, as indicated by the rates of subjects screening positive for depression (37.1\%) and for anxiety (26.9\%), as well as the high mean scores on both screening instruments. We did not evaluate the specificity of screening, but the central decision to refer or not to refer for treatment after a review by the mental health team comprised a de facto validation. For example, while $37.1 \%$ of screened subjects scored in the positive range for depression, only $31.5 \%$ of the total number of subjects who were screen-positive for depression and/or anxiety were referred for treatment. This small percentage of treatment referrals could reflect lack of specificity of the screening but might alternatively have resulted from the team's selectivity in making referrals; that is, only patients who appeared likely to adhere to and benefit from treatment were referred. Also, the low referral rate did not reflect the fact that a rudimentary mental health team had been in place before the initiation of the project, and some of the PCPs had continued to make referrals without prior screening. In addition, a substantial percentage of screen-positive subjects had already started treatment; of these subjects, those who had not yet experienced significant clinical improvement were then captured by the screening process as positive for depression and/or anxiety. On the other hand, there was a surprisingly high rate of screen-negative referrals for treatment, $11.8 \%$, a finding we regarded as consistent with the emphasis on clinical judgment in the referral process in lieu of reliance on screening data alone.

The referral process proved to be clinically justifiable as well as selective. Without following a specific protocol, we found that the patients referred for treatment were, based on screening scores and chart review, clinically symptomatic and that the interventions involving medications were assigned to those with the most severe anxiety and/or depression. Also, patients referred to "Social Services Intervention Only" may have been experiencing a financial or housing problem that needed to be addressed by a social worker but were not necessarily depressed or anxious.

The logistic regression models revealed that subjects who screened positive for depression, anxiety, or both were older, more likely to be female, and more often lived alone than screen-negative subjects. An association between living alone, which we viewed as a proxy indicator for social isolation, and screening positive for depression and/or anxiety became apparent in several steps of the data analysis. Living alone was first an independent predictor of screening positive in the logistic regression models. Then, living alone and referrals related to living alone as documented on the needs assessment emerged as predictors of screening positive for anxiety and/or depression.

One of the most important findings was the considerable prevalence (approximately 10\%) of suicidality, broadly comparable to the $7.3 \%$ figure found in a mixed-age Veterans Administration outpatient setting [26]. While this rate includes the full range of scores, including the least frequent suicidal thoughts, and while there were no suicides or suicide attempts among subjects screened during the study period, this finding points to the potential for such events and the need for vigilance in an age demographic having the highest population-based suicide rates in the United States. [27]. Although geriatric suicide has been closely linked to depression [28], an earlier study also conducted in New York City found that only a small proportion of elderly suicide victims had postmortem evidence of antidepressant medication, implicating systemic failures in delivering depression treatment to older adults [29]; in fact, the link between such failures of treatment delivery and geriatric suicide has been one of the chief rationales for the development of CCMs [7].

4.2. PASSE-PC and Other CCMS. In this paper we describe a straightforward, uncomplicated model of integrating mental health services into a university-based geriatric ambulatory practice. To date, several other CCMs integrating geriatric mental health care into primary care settings have been introduced. One of these, the TIP (Treatment Initiation Program) was studied partly in our own ambulatory care practice. In TIP, barriers to adherence to antidepressant medication treatment were identified and addressed by mental health personnel, augmenting the efforts of PCPs and psychiatric prescribers [30].

The CCM described here, PASSE-PC, mirrors the previous models in its core features. All, including PASSE-PC, featured interdisciplinary problem-solving, whereby PCPs 
and mental health professionals worked together to address patients' psychiatric conditions. For all of the models except PROSPECT (Prevention of Suicide in Primary Care Elderly Collaborative Trial) [31], in which mental health nurses were deployed to physicians' offices in the community in an advisory capacity, direct psychiatric services were made available on-site at the primary care solo or group practices. This innovation, referred to as "co-location," improves access to mental health services because of physical proximity; then, since the setting is already familiar to the patients, colocation also serves to increase their comfort with psychiatric treatment. The collaborative process in all of the models was further enhanced by greater opportunities for in-person communication between PCPs and mental health providers.

Like PROSPECT and IMPACT (Improving Mood Promoting Access to Collaborative Care Treatment) [14], psychotherapy in PASSE-PC was based upon PST (ProblemSolving Therapy). This intervention has been shown to be effective for elderly primary care patients with depression [23]. In addition, the sample size of the present study $(N=$ 1505) was comparable to those of the introductory trials of other CCM investigations: PRISM-E (Primary Care Research in Substance Abuse and Mental Health for the Elderly [32]) $(N=2002)$; IMPACT $(N=1801)$; and PROSPECT $(N=$ 599). However, unlike PRISM-E, IMPACT, PROSPECT, and TIP, the initial development and trial of PASSE-PC was not a randomized study, nor, as in PRISM-E, PROSPECT and TIP were multiple sites used.

Among the strengths of the PASSE-PC model were its simplicity, ease of implementation, and its emphasis on systematic screening as the principal method of case-finding. With respect to the last feature, the PHQ-9 and GAD-7 questionnaires have been extensively validated [17-22] and are well suited for screening large numbers of patients. These tests are brief and usable for individuals of all educational levels and also for those with mild to moderate cognitive impairment.

Moreover, the PASSE-PC model, unlike some of the other CCMs, made use of existing clinical personnel. The cost of implementation was low, with the most expensive line, that of the geriatric psychiatrist, required on only a part-time basis; full-time lines for the clinical social worker and psychiatric nurse practitioner were already in place. The modest cost in turn facilitated a smooth fit with the center's infrastructure. Although this trial involved a single, unique site, and nonacademic settings might not be expected to have preexisting lines for clinical social workers, nurse practitioners, or a psychiatrist, the efficiency of the overall program nevertheless suggested a broad adaptability to primary care group practices similar in size and scale to the one described here. At the Wright Center, the relatively low cost outlay also permitted us to follow subjects for up to 48 months, longer than the other models, and to allot time for medical education, both of these features suggesting a potential for sustainability. Ultimately it is hoped that primary care physicians, at our Center or elsewhere, will feel increasingly competent to diagnose and manage elderly patients with depression or anxiety, reserving psychiatric referral for the most complicated cases.
Given the paucity of geriatric psychiatrists in the United States [33], specialty care of elderly patients with mood disorders and other psychiatric syndromes is seriously scarce. This is a problem that cannot be addressed on a public health scale without involving primary care practitioners. It follows that treatment must be made accessible in settings where symptomatic elderly patients are most likely to be identified and most amenable to intervention.

4.3. Limitations of the Study. The principal limitations centered on the screening process. Although screening was the central method of psychiatric case-finding in PASSE-PC, it was in fact restricted in scope, eliciting symptoms, not diagnoses, and focusing solely on depression and anxiety; however, psychiatric and medical comorbidities, drug and alcohol abuse, and functional status were considered in all of the mental health team's assessments and referral deliberations. Also, because the study was carried out in a busy medical practice without dedicated research personnel, the distribution of questionnaires, and the recording of data devolved mainly upon clinicians whose principal responsibilities lay elsewhere. Screening was extensive but therefore neither ideally systematic nor complete, resulting in a convenience sample and variable quantities of missing data. Sensitivity and specificity of the screening process were not examined, and the mental health team did not establish reliability in referring subjects for treatments; moreover, the needs assessments were done by consensus rather than independently. Further, it was not known how many cases would have been identified and referred for treatment without screening. Lastly, the comparatively affluent and predominantly non-Hispanic white study population might raise questions of generalizability.

\section{Conclusions}

A new collaborative care model for screening, case-finding, and treatment of depression and anxiety in a geriatric primary care practice was found to be both clinically meaningful and feasible. The results from initial screening identified age, female gender, and living alone as predictors of depression and anxiety and also revealed a high frequency of suicidal ideation. In the future, studies comparing the findings of different teams working within a single setting would help to confirm the results reported here. It might also be useful to compare the experience of teams relying on screening instruments with teams using clinical referrals from primary care physicians in the usual manner. Studies that quantify known correlates and modifiers of geriatric affective symptoms such as functional status, cognitive impairment, and drug and alcohol abuse would be of additional value.

\section{Abbreviations}

CCMs:

PASSE-PC:
Collaborative care models

Psychiatric Assessment and

Screening for the

Elderly-Primary Care
(New York State) OMS: Office of Mental Health 
PHQ-9: $\quad$ Patient Health Questionnaire-9

GAD-7: $\quad$ Generalized Anxiety Disorder-7

PCP: $\quad$ Primary Care Physician

NP: $\quad$ (Psychiatric) Nurse practitioner

SW: $\quad$ (Clinical) Social Worker

PST: $\quad$ Problem-Solving Therapy

ANOVA: Analysis of Variance

MANOVA: Multivariate analysis of variance

ADLs: $\quad$ Activities of daily living

TIP: $\quad$ Treatment Initiation Program

PROSPECT: Prevention of Suicide in Primary

Care Elderly Collaborative Trial

IMPACT: Improving Mood Promoting

Access to Collaborative Care

Treatment

PRISM-E: Primary Care Research in

Substance Abuse and Mental

Health Screening for the Elderly.

\section{Conflict of Interests}

None of the authors have competing interests to report.

\section{Authors' Contribution}

Robert C. Abrams directed the design of the study, participated in the analysis and interpretation of data, and wrote the original draft of the paper. Blanca Boné codirected the design of the study, participated in the analysis and interpretation of data, and contributed to the preparation of the paper. M. Cary Reid supervised the design and data analysis phases of the project and contributed significantly to the writing of the paper. Ronald D. Adelman guided the research strategy and implementation of the data collection, contributed to data analysis, and also participated in the writing and editing of the paper. Risa Breckman worked with Ronald D. Adelman in developing the overall research strategy and design of the project. Ronald Goralewicz led the mental health team and designed the research procedures for subjects' clinical assessments and referral to treatment. Ronald Goralewicz also managed the on-site data collection. Marlena Palombo led the social work contributions to both the research effort and clinical interventions. Marlena Palombo also organized, directed, and maintained the study's data base. Amy Stern designed the social work contributions to the research and clinical aspects of the program and was succeeded in this role by Marlena Palombo. Amy Stern also contributed to the maintenance of the study's data base. Rouzi Shengelia coordinated the roles of all participants in the project and contributed significantly to the writing and preparation of the paper. Jeanne Teresi supervised all aspects of data analysis and interpretation and also contributed significantly to the writing and editing of the final paper.

\section{Acknowledgments}

To varying degrees, each of the authors received salary support from the New York State Office of Mental Health, which underwrote the project involving Weill Cornell and other institutional groups in New York. Both Drs. Abrams and Reid received support from the Howard and Phyllis Schwartz Philanthropic Fund. Dr. Reid was also supported by the National Institute on Aging (P30AG022845).

\section{References}

[1] J. J. Gallo and B. D. Lebowitz, "The epidemiology of common late-life mental disorders in the community: themes for the new century," Psychiatric Services, vol. 50, no. 9, pp. 1158-1166, 1999.

[2] D. C. Steffens, G. G. Fisher, K. M. Langa, G. G. Potter, and B. L. Plassman, "Prevalence of depression among older Americans: the aging, demographics and memory study," International Psychogeriatrics, vol. 21, no. 5, pp. 879-888, 2009.

[3] A. L. Byers, K. Yaffe, K. E. Covinsky, M. B. Friedman, and M. L. Bruce, "High occurrence of mood and anxiety disorders among older adults: the National Comorbidity Survey Replication," Archives of General Psychiatry, vol. 67, no. 5, pp. 489-496, 2010.

[4] I. R. Katz, P. A. Parmelee, and J. E. Streim, "Depression in older patients in residential care: significance of dysphoria and dimensional assessment," The American Journal of Geriatric Psychiatry, vol. 3, no. 2, pp. 161-169, 1995.

[5] E. F. Chaney, L. V. Rubenstein, C.-F. Liu et al., "Implementing collaborative care for depression treatment in primary care: a cluster randomized evaluation of a quality improvement practice redesign," Implementation Science, vol. 6, no. 1, article 121, 2011.

[6] S. Gilbody, T. Sheldon, and A. House, "Screening and casefinding instruments for depression: a meta-analysis," $C M A J$, vol. 178, no. 8, pp. 997-1003, 2008.

[7] M. L. Bruce, T. R. Ten Have, C. F. Reynolds III et al., "Reducing suicidal ideation and depressive symptoms in depressed older primary care patients: a randomized control trial," Journal of the American Medical Association, vol. 291, no. 9, pp. 1081-1091, 2004.

[8] P. J.van't Veer-Tazelaar, H. W. J.van Marwijk, P. van Oppen et al., "Stepped-care prevention of anxiety and depression in late life: a randomized controlled trial," Archives of General Psychiatry, vol. 66, no. 3, pp. 297-304, 2009.

[9] P. J. van't Veer-Tazelaar, H. W. J. van Marwijk, P. van Oppen et al., "Prevention of late-life anxiety and depression has sustained effects over 24 months: a pragmatic randomized trial," The American Journal of Geriatric Psychiatry, vol. 19, no. 3, pp. 230239, 2011.

[10] G. J. Kennedy, "Should primary care be the primary site of geriatric mental health care?" The American Journal of Geriatric Psychiatry, vol. 13, no. 9, pp. 745-747, 2005.

[11] K. Pollock and J. Grime, "Patients' perceptions of entitlement to time in general practice consultations for depression: qualitative study," British Medical Journal, vol. 325, no. 7366, pp. 687-690, 2002.

[12] J. D. Lish, M. A. Kuzma, D. T. Lush, G. Plescia, N. J. Farber, and M. Zimmerman, "Psychiatric screening in primary care: what do patients really want?" Journal of Psychosomatic Research, vol. 42, no. 2, pp. 167-175, 1997.

[13] C. Koyanagi, Get It Together: How to Integrate Physical and Mental Health Care for People with Serious Mental Disorders, Bazelon Center for Mental Health Law, Washington, DC, USA, 2004. 
[14] E. M. Hunkeler, W. Katon, L. Tang et al., "Long term outcomes from the IMPACT randomised trial for depressed elderly patients in primary care," The British Medical Journal, vol. 332, no. 7536, pp. 259-262, 2006.

[15] M. Schoenbaum, J. Unützer, C. Sherbourne et al., "Costeffectiveness of practice-initiated quality improvement for depression: results of a randomized controlled trial," Journal of the American Medical Association, vol. 286, no. 11, pp. 1325-1330, 2001.

[16] S. Gilbody, P. Bower, and P. Whitty, "Costs and consequences of enhanced primary care for depression: systematic review of randomised economic evaluations," British Journal of Psychiatry, vol. 189, pp. 297-308, 2006.

[17] S. Gilbody, D. Richards, S. Brealey, and C. Hewitt, "Screening for depression in medical settings with the Patient Health Questionnaire (PHQ): a diagnostic meta-analysis," Journal of General Internal Medicine, vol. 22, no. 11, pp. 1596-1602, 2007.

[18] S. Gilbody, D. Richards, and M. Barkham, "Diagnosing depression in primary care using self-completed instruments: UK validation of PHQ-9 and CORE-OM," British Journal of General Practice, vol. 57, no. 541, pp. 650-652, 2007.

[19] K. Kroenke, R. L. Spitzer, and J. B. W. Williams, "The PHQ9: validity of a brief depression severity measure," Journal of General Internal Medicine, vol. 16, no. 9, pp. 606-613, 2001.

[20] K. Kroenke and R. L. Spitzer, "The PHQ-9: a new depression diagnostic and severity measure," Psychiatric Annals, vol. 32, no. 9, pp. 509-515, 2002.

[21] K. Kroenke, R. L. Spitzer, and J. B. W. Williams, "The patient health questionnaire-2: validity of a two-item depression screener," Medical Care, vol. 41, no. 11, pp. 1284-1292, 2003.

[22] R. L. Spitzer, K. Kroenke, J. B. W. Williams, and B. Löwe, "A brief measure for assessing generalized anxiety disorder: the GAD7," Archives of Internal Medicine, vol. 166, no. 10, pp. 1092-1097, 2006.

[23] G. S. Alexopoulos, P. Raue, and P. Areán, "Problem-solving therapy versus supportive therapy in geriatric major depression with executive dysfunction," The American Journal of Geriatric Psychiatry, vol. 11, no. 1, pp. 46-52, 2003.

[24] S. Samuels, R. C. Abrams, R. Shengelia et al., "Integration of geriatric mental health screening into a primary care practice: a patient satisfaction survey," International Journal of Geriatric Psychiatry, 2014.

[25] S. Samuels, R. Breckman, E. Woods et al., "Integration of mental health screening into primary care: are patients satisfied?" Journal of the American Geriatrics Society, vol. 58, pp. s168-s169, 2010.

[26] J. D. Lish, M. Zimmerman, N. J. Farber, D. T. Lush, M. A. Kuzma, and G. Plescia, "Suicide screening in a primary care setting at a Verterans Administration medical center," Psychosomatics, vol. 37, pp. 413-424, 1996.

[27] Y. Conwell, P. R. Duberslein, C. Cox, J. Herrmann, N. Forbes, and E. D. Caine, "Age differences in behaviors leading to completed suicide," The American Journal of Geriatric Psychiatry, vol. 6, no. 2, pp. 122-126, 1998.

[28] W.-H. Kuo, J. J. Gallo, and W. W. Eaton, "Hopelessness, depression, substance disorder, and suicidality-a 13-year community-based study," Social Psychiatry and Psychiatric Epidemiology, vol. 39, no. 6, pp. 497-501, 2004.

[29] R. C. Abrams, A. C. Leon, K. Tardiff, P. M. Marzuk, C. Li, and S. Galea, "Antidepressant use in elderly suicide victims in New York City: an analysis of 255 cases," Journal of Clinical Psychiatry, vol. 70, no. 3, pp. 312-317, 2009.
[30] J. A. Sirey, M. L. Bruce, and G. S. Alexopoulos, "The treatment initiation program: an intervention to improve depression outcomes in older adults," The American Journal of Psychiatry, vol. 162, no. 1, pp. 184-186, 2005.

[31] Y. Bao, G. S. Alexopoulos, L. P. Casalino et al., "Collaborative depression care management and disparities in depression treatment and outcomes," Archives of General Psychiatry, vol. 68, no. 6, pp. 627-636, 2011.

[32] S. J. Bartels, E. H. Coakley, C. Zubritsky et al., "Improving access to geriatric mental health services: a randomized trial comparing treatment engagement with integrated versus enhanced referral care for depression, anxiety, and at-risk alcohol use," The American Journal of Psychiatry, vol. 161, no. 8, pp. 1455-1462, 2004.

[33] American Association for Geriatric Psychiatry, http://www .aagponline.org/. 


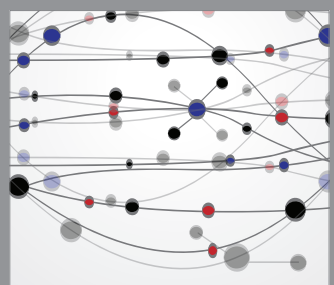

The Scientific World Journal
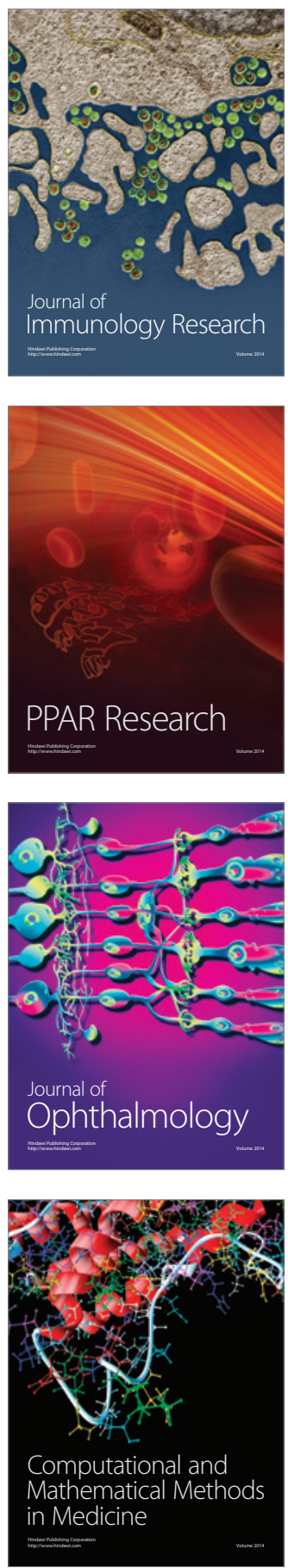

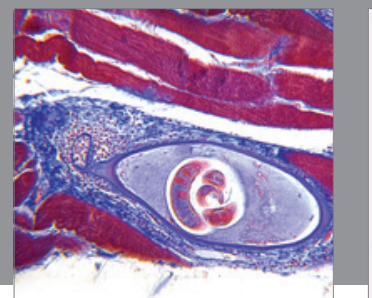

Gastroenterology

Research and Practice
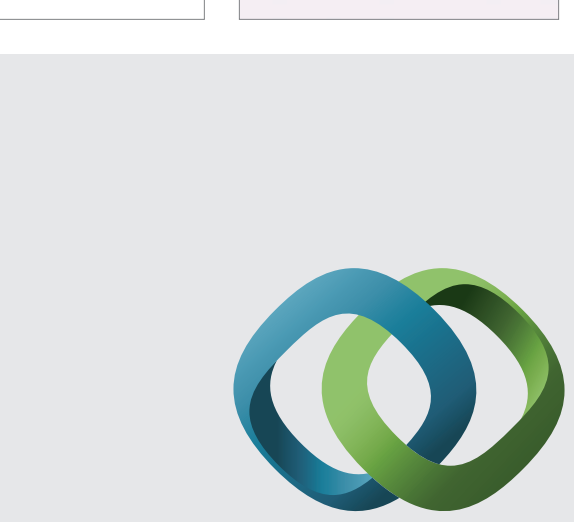

\section{Hindawi}

Submit your manuscripts at

http://www.hindawi.com
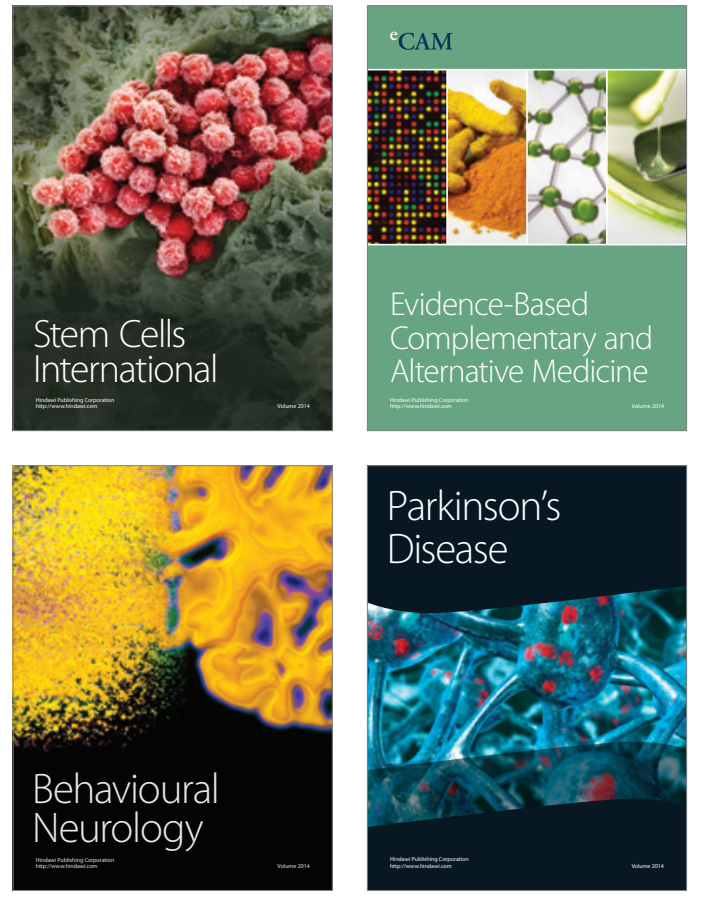
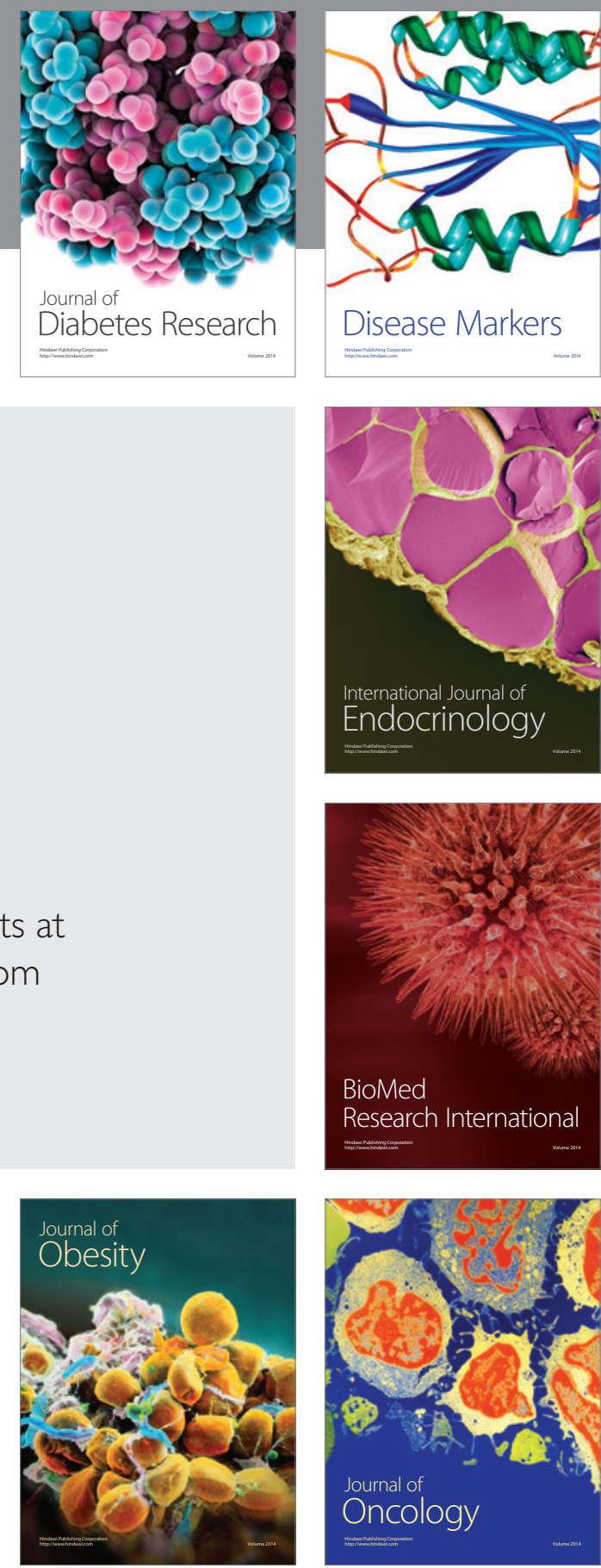

Disease Markers
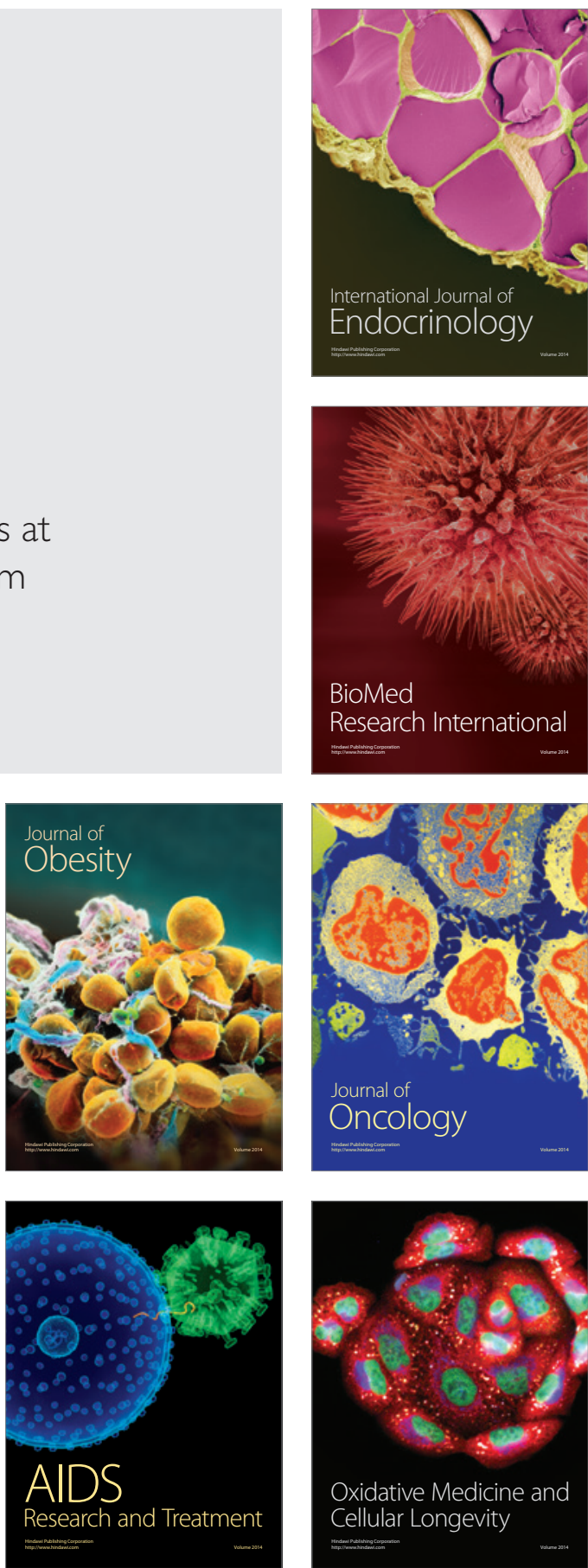